\title{
Acción colectiva para la regularización dominial. Análisis de la experiencia del barrio El Molino de La Plata
}

\section{Collective action for dominial regularization. Analysis of the experience in the El Molino de La Plata neighborhood}

\author{
Andrea Di Croce Garay \\ andreadicroce@hotmail.com \\ Centro Interdisciplinario de Estudios Complejos. \\ Facultad de Arquitectura y Urbanismo. Universidad \\ Nacional de La Plata, Argentina
}

Recepción: 20 Julio 2020

Aprobación: 30 Septiembre 2020

Publicación: 03 Mayo 2021

Cita sugerida: Di Croce Garay, A.(2021). Acción colectiva para la regularización dominial. Análisis de la experiencia del barrio El Molino de La Plata. Geograficando, 17(1), e093. https://doi.org/10.24215/2346898Xe093

\begin{abstract}
Resumen: En los procesos de transformación de las condiciones habitacionales de los barrios populares inciden diversos actores y factores, lo que permite analizarlos desde múltiples dimensiones. En este trabajo se propone indagar en la dimensión colectiva de esos procesos, buscando reconocer las especificidades que aporta al mejoramiento del hábitat. Para ello se acude al estudio de un caso, en el que se tuvo por objetivo la regularización dominial de un barrio surgido de una ocupación de tierras. El trabajo fue realizado a partir de entrevistas semiestructuradas, participación en asambleas barriales y recorridos por el barrio, actividades complementadas con el análisis de publicaciones de las organizaciones sociales intervinientes y datos de distintos registros públicos. El análisis permitió registrar la relevancia de la acción organizada en el acceso a la regularización dominial (así como al mejoramiento de distintos aspectos habitacionales), al tiempo que reconoce la necesaria voluntad estatal y política para el avance de estas operatorias.
\end{abstract}

Palabras clave: Regularización dominial, Acción colectiva, Barrios Populares, El Molino, La Plata.

Abstract: Various actors and factors affect transformation processes in the housing conditions of popular neighborhoods, which allows for an analysis on multiple dimensions. In this article, the aim is to investigate the collective dimension in these processes, seeking to recognize the specificities that it contributes to habitat improvement. To do this, a case study is used, in which the objective was to regularize a neighborhood that emerged from a land occupation. The study was carried out from semi-structured interviews, participation in neighborhood assemblies and tours of the neighborhood, complemented with the analysis of publications by intervening social organizations, and data from different public registries. The analysis made it possible to register the relevance of organized action in the access to property regularization, as well as improvement on different housing aspects, while recognizing the necessary State and political will for the advancement of these operations.

Keywords: Domain regularization, Collective action, Popular Neighborhoods, El Molino, La Plata. 


\section{INTRODUCCIÓN}

Un hormiguero. En permanente movimiento, en constante proceso de modificación, trazado por el trabajo colaborativo, cargado de esfuerzos monumentales, de rápida respuesta organizada ante los pisoteos desinteresados que destruyen lo avanzado. Con esta expresión podemos describir lo que cotidianamente sucede en la mayoría de los barrios populares para resolver desde el alimento hasta la vivienda.

La regularización dominial es también motivo de esos movimientos. Si bien estas operatorias implican indefectiblemente la acción estatal, pareciera que la movilización barrial representa un factor de relevancia en su desarrollo. Desde ese punto de partida, este artículo propone revisar los movimientos de hormiga que posibilitaron el comienzo de la regularización dominial en un barrio popular de La Plata: El Molino. Como parte del trabajo de tesis de Maestría ${ }^{1}$, el objetivo fue indagar en las características y los aportes que la acción colectiva brindó a ese proceso, a partir de asumir la relevancia que las acciones organizadas tienen en estos contextos.

El trabajo se organiza en tres apartados. En primer lugar, se indican aspectos metodológicos que permitieron desarrollar el estudio. Luego se presenta el estudio de caso, analizando el origen del barrio, el proceso de acción colectiva que permitió la adquisición del predio y la situación a la que se logró arribar, y los aportes que el carácter colectivo brindó al proceso. Por último, se presentan las reflexiones finales.

\section{Metodología}

Con el objetivo de contribuir a repensar posibles aportes que genera la dimensión colectiva de los procesos de regularización dominial en barrios populares, ${ }^{2}$ se definieron objetivos específicos que condujeron el análisis de caso: (i) reconstruir las estructuras organizativas utilizadas por lxs habitantes del barrio, (ii) caracterizar los vínculos de lxs vecinxs con organizaciones sociales y políticas, (iii) analizar las relaciones logradas (o no) en el proceso de regularización dominial entre las familias organizadas y organizaciones sociales con diferentes estamentos del Estado, (iv) analizar si las dinámicas asociadas con la regularización dominial derivaron en la gestación o en el fortalecimiento de otros procesos barriales.

La estrategia metodológica combinó el uso de técnicas de los métodos cualitativos con algunas de los cuantitativos. Para la caracterización de la situación habitacional, se utilizaron diversas fuentes secundarias de acceso público: Censo Nacional de Población, Hogares y Vivienda 2010, Registro Público Provincial de Villas y Asentamientos Precarios (RPPVAP), Registro Nacional de Barrios populares (ReNaBaP). También se indagó en las páginas web y redes sociales de las organizaciones sociales intervinientes.

A su vez, se realizaron entrevistas exploratorias a trabajadorxs y extrabajadorxs de la ex Subsecretaría Social de Tierra Urbanismo y Vivienda (SSTUV), ${ }^{3}$ dos entrevistas semi-estructuradas a referentes del Frente de Organizaciones en Lucha (FOL) y una entrevista colectiva a tres referentes barriales. También se participó en una asamblea barrial y se realizó un recorrido por el barrio junto a referentes de la asamblea. Para acceder a estos referentes se utilizaron estrategias como contactos previos, pedidos formales a las organizaciones participantes, llamados telefónicos, etc. Asimismo, se utilizó la técnica conocida como "bola de nieve", que consiste en utilizar las redes personales de los contactos iniciales para acceder progresivamente a otros informantes (Piovani, 2007).

A partir de esta tarea y de la revisión bibliográfica, se caracterizaron los procesos mediante los que el barrio inició su proceso de regularización dominial, apelando a la tipología de actores retomada de Herzer y Pírez (1993; 1995): actores estatales (oferentes de programas habitacionales, subsidios y asistencia técnica y social; son incorporadas en esta categoría las fuerzas de seguridad), actores económicos (mediante el mercado participan en la subdivisión y venta de suelo y/o la construcción y venta de viviendas), actores comunitarios (encarnados en lxs propixs afectadxs de las demandas habitacionales, en conjunto o no con organizaciones 
sociales; aportando a veces solidaria y colectivamente su trabajo para la construcción de viviendas y la urbanización de barrios).

\section{Resultados}

A partir de las indagaciones realizadas, presentamos a continuación cuatro apartados en los que se recorren aspectos referidos a: (1) el origen del barrio, (2) el proceso de acción colectiva que permitió la adquisición del predio de El Molino, (3) la situación a la que se logró arribar, (4) análisis de los aportes de la acción colectiva.

\section{Origen del barrio: ocupar tierras ociosas como solución habitacional}

Durante las primeras aproximaciones al trabajo de campo, había escuchado hablar del barrio El Molino y del barrio Yuelito. Pero cuando buscaba estos nombres en el Registro Nacional de Barrios Populares (RENABAP), no los encontraba. A su vez, la dirección a la que se aludía en los relatos figuraba ${ }^{4}$ en ese Registro como parte del barrio Joel, pero ese nombre no aparecía en ninguna de mis anotaciones. Bastó con ir a una asamblea barrial de El Molino para revelar el misterio: en el relevamiento de barrios populares se registró el predio comprendido entre las calles 1 a 118 y 609 a 613 como barrio "Joel”, pero en la realidad son dos barrios, uno desprendimiento del otro.

Los barrios Yuelito (comprendido entre las calles 1, 117, 609 y 613) y El Molino (ubicado entre las calles $117,118,609$ y 613 ) se encuentran en Villa Elvira, una de las 18 delegaciones de la ciudad de La Plata (Figura 1). Ubicada en el sudeste del partido, es junto con Melchor Romero la delegación con mayor cantidad de asentamientos populares informales (API) (RPPVAP, 2015). Más del $60 \%$ de los asentamientos de esta delegación surgieron a partir del año 2001.

\section{FIGURA 1}

Emplazamiento del barrio El Molino

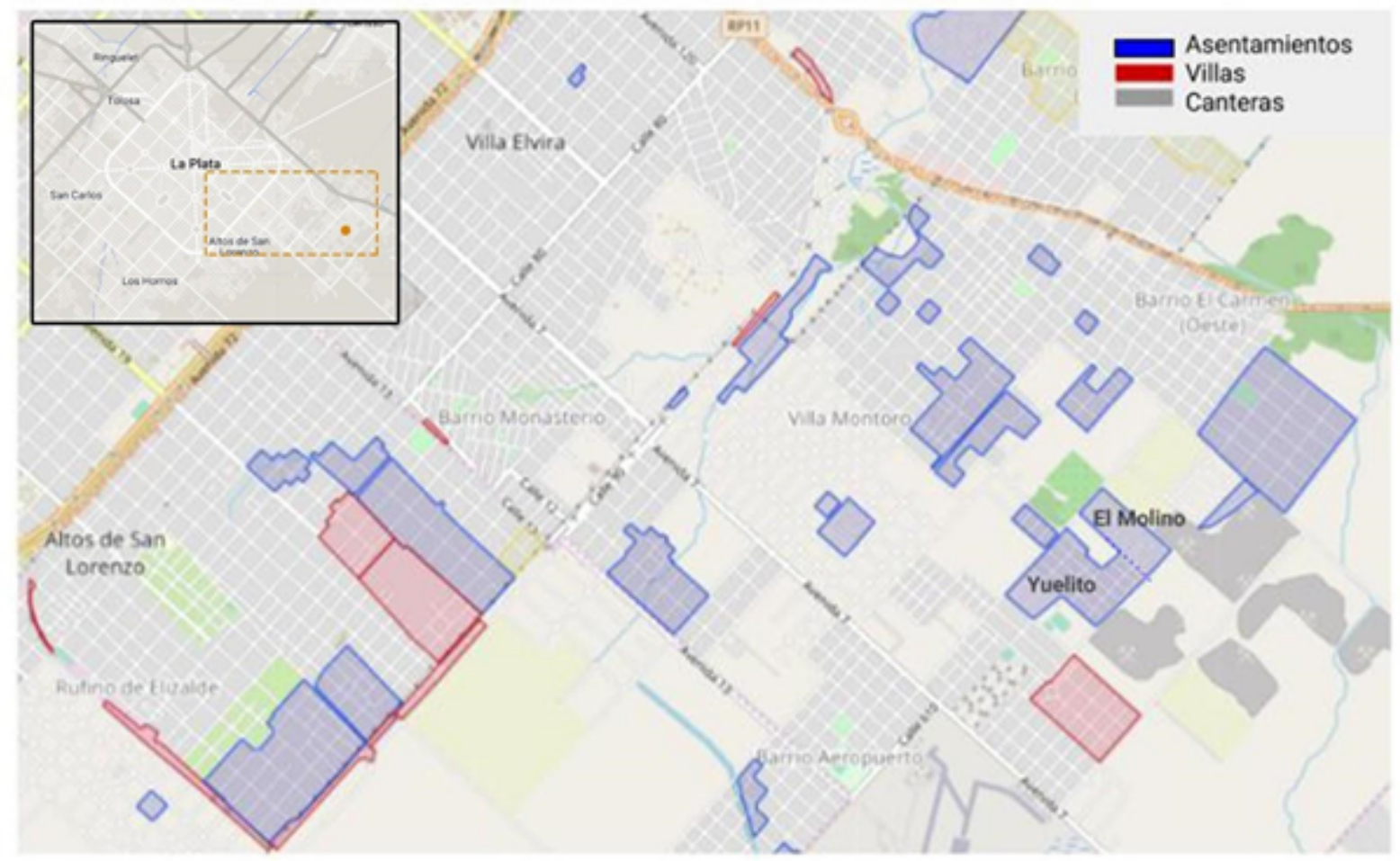


Luego de la crisis económica argentina del 2001, el boom inmobiliario había comenzado a remodelar el casco histórico de La Plata. Ese proceso -consecuencia del descreimiento en las instituciones bancarias después de la crisis de diciembre de 2001 y el consecutivo proceso de "inversión en ladrillo" (Del Río y González, 2018)- había atraído a una fuerte cantidad de migrantes dispuestos a trabajar en la construcción. Imposibilitadxs de resolver su acceso a la vivienda en contextos de formalidad urbana, 5 se fueron ubicando en distintos barrios populares de la periferia local o fueron ocupando predios vacantes, tal como sucedió en la fundación de La Plata, cuando el proceso de construcción del centro de la ciudad fue acompañado de procesos de urbanización de tierras en la periferia. El barrio Yuelito en Villa Elvira fue uno de los barrios que desde el año 2004 albergó a varias de estas familias. En este barrio, durante el año 2011 el Movimiento 7 de abril (hoy Frente de Organizaciones en Lucha, FOL) había empezado a conformar el área de Hábitat y Vivienda, desde la que trabajaba en asambleas con la regularización urbanística y dominial. Durante una de estas asambleas, un grupo de vecinxs se acercó a un dirigente del Movimiento para avisar que en los próximos días entrarían a ocupar el predio lindero que se encontraba vacante. Desde hacía tiempo, en Yuelito se empezaban a presentar graves problemas de hacinamiento, producto de desdoblamientos y crecimientos intrafamiliares. Simultáneamente, la demanda habitacional de muchas familias migrantes residentes en La Plata continuaba sin respuestas: muchxs de lxs migrantes que habían sido atraídos por la posibilidad laboral en las construcciones habían atravesado para entonces un derrotero de peregrinaciones por distintos barrios y ocupaciones de tierras. Expresiones como "Vivíamos en un alquiler, desde que llegamos acá estamos buscando un lugarcito, porque alquilar te lleva mucho", "Casi siempre estuve en busca de un espacio para construir mi vivienda, vivíamos en alquiler y no sobraba nada" 6 dan cuenta de las dificultades de esta población para resolver su situación habitacional. Las diez hectáreas vacantes frente a Yuelito parecían ofrecer una solución.

El domingo 9 de octubre de 2011, dos semanas antes de las elecciones presidenciales, 20 familias entraron al predio a las 6:00 a.m. A las 11:00 eran 160 familias, y a la tarde había lista de espera. Lo que hasta hacía pocos años no había sido más que tierras vacantes rodeadas por canteras comenzaba a transformarse en el barrio El Molino. Y lo que podría haber sido una historia más de desalojo por ocupación de tierras, logró torcer el viento y convertirse en un proceso organizado de producción de hábitat, referente para muchos otros procesos $^{7}$. Pero para torcer ese viento harían falta algunxs actores, varios aportes y determinadas acciones, en las que indagaremos a continuación.

\section{Adquisición del predio: de la ocupación a la venta, de la venta a la urbanización organizada}

La ocupación del predio del barrio El Molino desencadenó una serie de acciones que involucraron a diversos actores. En este proceso, a diferencia de otras ocupaciones ${ }^{8}$, el diálogo entre actorxs fue una de las principales herramientas a través de la que se encontraron respuestas a las distintas necesidades y objetivos de lxs diversxs actorxs (Figura 2):

- Frente de Organizaciones en Lucha (FOL). Organización social de base territorial, con objetivo de acompañar la organización popular.

- Ocupantes: cuya necesidad se vincula con resolver una necesidad habitacional. Organizadxs en asamblea vecinal.

- Propietarixs: el predio estaba en venta desde hacía 10 años. Pertenecía a una familia local.

- Organizaciones de técnicxs: aportan los saberes técnicos a procesos de organización barrial.

- Estado: participa en el proceso mediante la intervención de algunas de sus instituciones: SSTUV, policía. Escribanía general de Gobierno. 
FIGURA 2

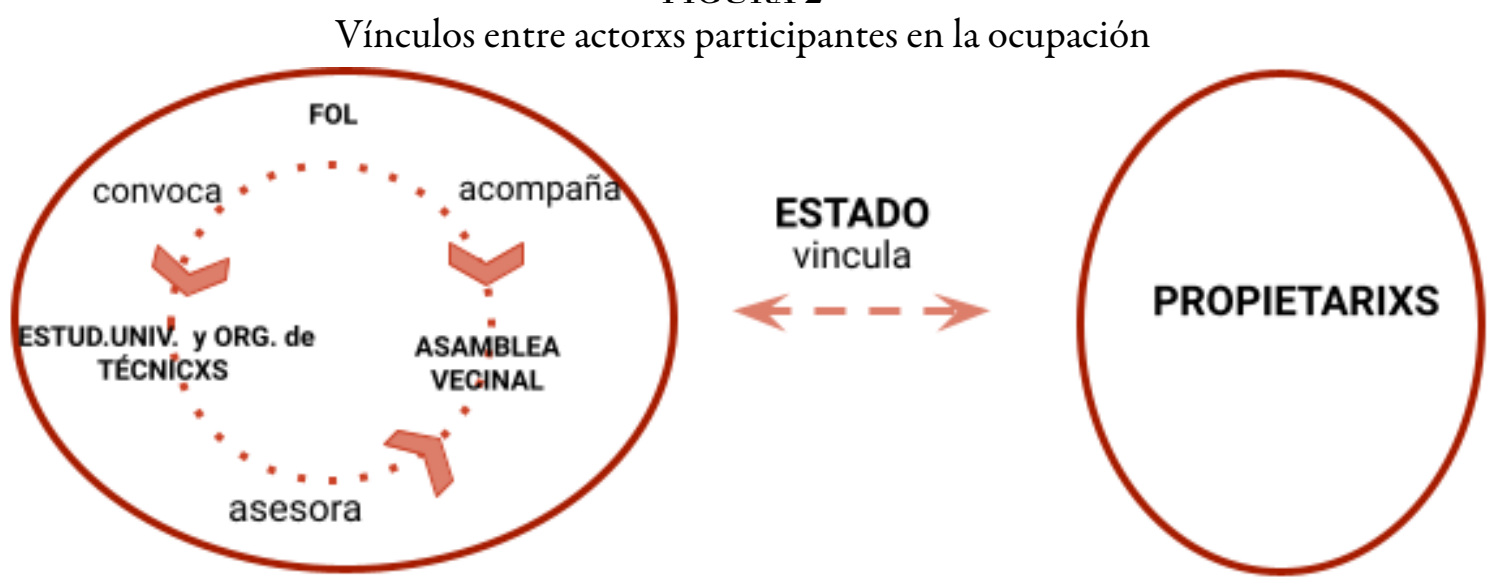

Elaboración propia

Ante el aviso de que se ocuparía el predio, desde la organización social se decidió acompañar el proceso:

"Nos habíamos enterado de que se iba a tomar un predio en barrio Aeropuerto, y en primera instancia es como que bueno, fueron algunos compañeros como para apoyar esa situación, o bueno... para organizar en algún punto". (Integrante del Movimiento 7 de abril)

Ese acompañamiento fue relevante para la inmediata conformación de la asamblea de El Molino. Al igual que el barrio, la asamblea fue un desprendimiento del barrio Yuelito: en un primer momento funcionó en una casa de ese barrio y luego en "el eucalipto" del nuevo barrio. Organizadxs mediante un cuerpo de delegadxs por manzana, se fueron abordando las distintas situaciones y problemas surgidos. Por un lado, los rumores de "re-toma" (más que los de desalojo) llevaron a organizar grupos de guardia en el predio. Desde la asamblea se organizaron turnos con el objetivo de cuidar el predio entre las personas que estaban en el listado "para contrato", es decir, entre quienes ya tenían un lote asignado por la asamblea vecinal. Por otro lado, la lista de espera que rápidamente se formó demandó respuestas. En relación con las dinámicas de lxs ocupantes con los terrenos (encontramos a quienes ocuparon y se quedaron, ocuparon y vendieron inmediatamente por falta de recursos, ocuparon con fines especulativos, ocuparon y lo cedieron a un familiar o conocido), se fue acordando en asamblea si se aceptaban o no cada uno de los ingresos surgidos de re-ventas o cesiones. Una estudiante de Arquitectura señalaba:

"No se vio demasiado querer sacar provecho con los punteros y esas cuestiones. La organización política sirvió para visualizar la existencia de punteros que quieran beneficiarse con el tema de la venta posterior de las tierras. La misma asamblea los iba expulsando". (V., estudiante de Arquitectura e integrante del FOL)

Si bien el predio se encontraba en venta, la ocupación podía considerarse beneficiosa para lxs (por entonces) propietarixs, pues mediante la negociación tenían la posibilidad de finalmente venderlo. Sin embargo, su primera reacción fue denunciar y acompañar el pedido de desalojo: pocas horas después de haber ingresado al predio, se presentaron integrantes de las fuerzas policiales con una supuesta orden de desalojo. Su llegada coincidió con la de integrantes de "La Ciega", colectivo de estudiantes y profesionales de Ciencias Jurídicas de la UNLP, que había sido contactado por el Movimiento 7 de Abril. Ante el intento de desalojo, el colectivo brindó su asesoramiento y mostró que la orden de desalojo era falsa. A través del asesoramiento del colectivo supieron que "el papel que nos trajo la gorra mientras tomábamos las 10 hectáreas, pese a lo que decían, era un chamuyo y no llegaba a ser una orden de desalojo" (tomado del Facebook de FOL). ${ }^{11}$

La segunda reacción de lxs propietarixs fue, entonces, acercarse a dialogar, acompañadxs por la policía. El estado de organización vecinal, acompañado del asesoramiento de la organización social y el colectivo de abogadxs, permitió proponer una compra colectiva de las tierras, que fue aceptada por lxs propietarixs. La 
intervención estatal incorpora en este punto otros actores además de la policía. En primer lugar, la SSTUV comenzó a participar en la mediación entre ocupantes y propietarixs, y coordinó un censo realizado por trabajdorxs sociales, necesario para la organización del cobro de las cuotas. La negociación parecía ir en marcha, pero el freno fue puesto por el propio Estado municipal: el precio acordado para la venta del terreno era "muy bajo" y podía desencadenar otras demandas de tierras a ese valor. Ante la dilatación de las tomas de decisiones, el Movimiento 7 de abril informó a la Municipalidad que, de no aprobarse la venta de tierras, cortarían todos los accesos a Villa Elvira el día de la elección presidencial, e imposibilitarían que lxs habitantes de la delegación participaran de los comicios. Tras ese aviso, en pocos días se definió entre las partes el precio final por lote, monto del cual el Estado municipal pondría un porcentaje. La venta estaba acordada.

Como se vio en el testimonio precedente, la colaboración de profesionales involucró también a estudiantes de arquitectura. Durante las negociaciones de venta del predio, el Movimiento percibió que el hecho de contar con un proyecto de urbanización del barrio podía posicionarlos en un lugar privilegiado. No resulta igual reclamar por un predio vacante que hacerlo para urbanizarlo de determinada forma. Una de las integrantes del Movimiento 7 de abril estudiaba en ese entonces en la Facultad de Arquitectura y Urbanismo de la UNLP, y tomó la tarea de proyectar el barrio junto a otra estudiante de la misma Facultad. Aunque todxs coincidían en no querer "hacer una villa", 12 la rápida distribución de lotes acercó la posibilidad de ocupar la tierra de forma "desordenada". Una de las estudiantes, refiriéndose al tema, señalaba:

"Por un lado estaba la organización de los vecinos, que decidan en asamblea, explicarles que era mejor que se diseñe el barrio antes de que todos vayan y se agarren un pedazo como siempre pasa, que estaba bueno que haya calles porque después iba a poder pasar el basurero, la ambulancia. Bueno, un montón de beneficios, que la toma de al lado no tiene."

Mediante cortas instancias participativas en asamblea, las dos estudiantes determinaron el programa de necesidades en el que basó el proyecto urbanístico (que contempló espacio para comedor y una huerta, espacios de producción, espacios recreativos, calles anchas, orientar las manzanas para que tengan buen asoleamiento) y confeccionaron las planillas para que lxs delegadxs relevaran las manzanas. El carácter participativo del proyecto permitió la apropiación de lxs vecinxs, quienes respetaron la mayor parte del proyecto a pesar del tiempo que llevó:

"A pesar de que se tardó, la gente respetó que había un proceso, que había gente diseñando. Se los hizo partícipes también porque cuando vieron los bocetos se pusieron recontentos."(V., estudiante de Arquitectura)

El molino emplazado sobre la calle 117 y 612 dio nombre al barrio. El predio fue finalmente loteado en 172 lotes, ubicados en 7 manzanas de 20 lotes y 4 manzanas de 8 lotes, con espacio para plaza y para el centro comunitario.

La distribución de los lotes fue por sorteo, aunque eso no evitó los problemas derivados de la preferencia de vivir sobre la calle 609 (por donde pasa el transporte público). La prioridad la tuvieron ancianxs y personas con distintas discapacidades, y luego quienes habían tenido mayor participación en la ocupación y en las actividades posteriores. Luego de la distribución, algunos lotes fueron intercambiados entre ocupantes.

\section{Situación a la que se arribó}

$\mathrm{Al}$ preguntarnos hasta dónde se llegó con la regularización dominial del barrio El Molino, las respuestas encontradas son diversas. Si bien en el acuerdo de venta la Escribanía General de Gobierno realizaría la escrituración social de los lotes, esta tarea no pudo ser realizada. Algunxs entrevistadxs indicaron que la escrituración no se finalizó por la falta de pago de la mayoría de sus vecinxs: un entrevistado mencionó que sólo pagaron 30 de lxs 172. Otra de las entrevistadas aseguró haber hecho relevamientos en el barrio en los que encontró que la mayoría sí había pagado, y señaló que según el abogado de la inmobiliaria el incumplimiento del acuerdo se debió a la falta de urbanización del barrio. Esta información, además de ser falsa, les incorpora 
a lxs habitantes del barrio la responsabilidad de urbanizar el barrio para que el Estado pueda escriturar. Otrxs entrevistadxs mencionan que para escriturar era necesario que desde el Concejo Deliberante se aprobara la rezonificación del predio; como ello no sucedió, se avanzó únicamente en la gestión de los boletos de compray venta. La multiplicidad de respuestas da cuenta en algún sentido de las dificultades para acceder a información por parte de lxs ocupantes.

Durante el año 2018, varixs vecinxs obtuvieron el certificado de vivienda otorgado por el RENABAP, mediante gestiones realizadas individualmente.

Más allá de la organización barrial que, aunque con variaciones, se mantuvo constante en el tiempo, con los años se ocuparon algunos sectores dentro del barrio: la plaza ubicada entre la calle 118 y la cantera, el extremo este de la tierra del sector comunitario, y la franja de 15 metros ubicada entre la calle 614 y la cantera.

Estas ocupaciones no son percibidas como parte del barrio El Molino, sino que son nombrados como "la villa" y "la toma". Si bien la mayoría de esas tierras estaban destinadas a espacios comunitarios, al momento de su ocupación no hubo reacción: no se expulsó a los ocupantes, pero tampoco se los ayudó. La decisión de inacción podría interpretarse como parte de las limitaciones de la organización vecinal. Sin embargo, encontramos en las entrevistas que fue parte de un acuerdo colectivo, vinculado a reconocer las necesidades de estos nuevos ocupantes con las que lxs mismxs habitantes de El Molino habían padecido.

\section{Aportes de las acciones colectivas}

En este apartado nos interesa indagar en los aportes de las acciones colectivas desarrolladas entre lxs diversos integrantes del entramado inter actoral de esta experiencia.

\section{Aportes desde "lo comunitario": la organización vecinal}

Así como en muchos asentamientos populares, en El Molino encontramos que un gran porcentaje de la población es de origen migrante, ya sea de otras provincias argentinas o de países limítrofes. El origen no resulta un dato menor: la tradición en la construcción, y en distintas formas de organización comunitaria que estas comunidades a veces traen consigo, fue retomada en los espacios de asamblea y en las estructuras a partir de las que se organizaron.

La existencia de reuniones de delegadxs y de asambleas barriales permitió ir dando respuestas a las situaciones conflictivas que se fueron presentando, como las ventas internas de terrenos, los cambios de lote, la "lista de espera", los intentos de re-toma.

En los meses que continuaron a la fecha de la ocupación, comenzaron a darse instancias de especulación inmobiliaria dentro de las tomas, que en algunos casos lograron ser abordadas desde los espacios comunitarios. Azuela de la Cueva (1999) sostiene que luego de la ocupación suele darse un proceso de configuración de un mercado. La acción colectiva, acompañada en estos casos por las organizaciones sociales, permitió abordar algunos aspectos de estas situaciones. Un integrante del FOL indicaba sobre el tema:

"A la hora de que algunas familias quieren vender la tierra, y se entienden como propietarixs individuales, se ponen en evidencia los diferentes niveles de debate y conciencia. Ante esto, la organización social tiene que poder intervenir y dar los debates posibles, y hasta promover la expulsión de las familias que rompen los acuerdos (y venden o alquilan la tierra ganada). El criterio que prima, el original de la toma, debe ser responder a la necesidad de tierra y vivienda."(P., integrante del FOL)

Con respecto a los rumores de "re-tomas", el problema encontró sus primeras respuestas en acciones colectivas, como guardias rotativas.

En lo que se refiere a la regularización dominial, en El Molino la venta directa con lxs propietarixs se dio durante el proceso de ocupación. Si bien no se puso en discusión que el objetivo último de la regularización dominial era el título individual de la tierra y la vivienda, se atravesaron situaciones colectivas con una 
búsqueda de resultados individuales, a partir de asumir la regularización dominial como problema de escala barrial.

Iniciados los trámites de regularización dominial, la existencia de cuerpos de delegadxs permitió, además del desarrollo de procesos democráticos de decisiones colectivas, garantizar la difusión de información entre las familias y el control interno de los pagos de las cuotas

\section{Aportes desde las organizaciones sociales}

En lo que refiere a organizaciones sociales, sus aportes fueron de gran relevancia. Desde días previos a la ocupación se avisó a integrantes del FOL que se tomarían los predios, y la organización decidió acompañar la ocupación. El primer aporte reconocido por lxs vecinxs entrevistadxs son las pautas sobre la estructura de delegadxs y funcionamiento de las asambleas.

Si bien la propuesta de comenzar un proceso de ocupación de tierras y/o regularización dominial partió de lxs vecinxs, los abordajes tuvieron particularidades propias derivadas, en parte, de las estructuras organizativas de las organizaciones intervinientes. En el caso del FOL, indicamos que contaba con una incipiente área de Hábitat, en la que participaban estudiantes de Arquitectura, y a través de la que se venían incorporando actividades ligadas a temáticas habitacionales. Además contaba con vínculos con organizaciones profesionales que alentaron y acompañaron el avance del proceso organizativo. Estos recursos condicionaron en gran medida los desenlaces.

Durante las negociaciones, las organizaciones sociales acercaron la posibilidad de difusión en medios alternativos (principalmente radios comunitarias) y el contacto con colectivos de técnicxs y con funcionarixs públicxs. A su vez, las experiencias de cada organización fueron transmitidas a las asambleas, y en algunos sentidos pudieron ser apropiadas. Lxs mismxs vecinxs del barrio indicaron, sin embargo, que para ellxs fue muy importante que lxs militantes estén presentes en las negociaciones porque "hablan mejor".

La trayectoria piquetera de la organización social generó, a su vez, aportes particulares: la capacidad de negociación del FOL fue un aporte significativo que puso en marcha las negociaciones a partir de anuncios de cortes de calles durante las jornadas electorales.

No podemos negar que los contextos políticos fueron condicionantes en cada uno de ellos (en el caso analizado, las cercanas elecciones presidenciales del año 2011). No obstante, el acompañamiento en la formación de cuerpos de delegadxs y asambleas, la coordinación con otras organizaciones sociales y profesionales, el vínculo con medios de comunicación, los repertorios de reclamo fueron aportes de las organizaciones sociales que tuvieron gran relevancia en los avances de la regularización dominial, que se complementaron con la necesaria voluntad política y los contactos directos con funcionarixs estatales.

\section{El aporte técnico}

En la experiencia encontramos intervenciones realizadas por estudiantes y graduadxs de la UNLP. Sus participaciones se dieron de distintas formas: desde colectivos profesionales, o convocadxs individualmente por integrantes de las organizaciones sociales. Tanto en sus presencias como en sus ausencias, podemos encontrar el valor de los aportes de técnicxs y profesionales especializadxs en problemas habitacionales durante conflictos territoriales.

Durante los procesos analizados, hubo instancias caracterizadas por la desinformación y/o por recibir múltiples respuestas a una misma consulta. La falta de asesoramiento y/o el desconocimiento técnico de integrantes de organizaciones sociales y vecinxs de los barrios llevó en algunas ocasiones a la dilatación de los procesos, así como a su inconclusión. En contraposición, encontramos las intervenciones realizadas por estudiantes y profesionales que se convirtieron en elementos relevantes para lxs ocupantes tanto en las 
negociaciones con lxs propietarixs y el Estado (la posibilidad de asesoramiento ante un desalojo, y el contar con un proyecto de urbanización, se convirtieron en herramientas de disputa en el proceso de negociación para la venta de la tierra), como en el proceso posterior de urbanización.

En varias de estas instancias reconocemos, asimismo, intercambios de saberes entre técnicxs y habitantes de los barrios. Por último, ante las ausencias de técnicos especialistas algunas situaciones se fueron resolviendo con los propios conocimientos de lxs habitantes de los barrios, los cuales, sin embargo, son muchas veces desvalorizados.

Cada uno de estos aportes logró acercar experiencias, métodos, saberes que complementaron los espacios de organización barrial y posibilitaron el fortalecimiento de los procesos de acceso a la tierra y regularización dominial, pero también pusieron en cuestión las relaciones entre universidades y/o profesionales y los problemas habitacionales.

\section{Trabajadorxs estatales y funcionarixs públicxs}

"Yo creo que el Estado en tu barrio es pura y exclusivamente acto del vecino. Porque no viene el Estado a tu barrio, nunca viene".

(M., vecina entrevistada)

Las primeras búsquedas al indagar en los vínculos establecidos con distintas jerarquías y jurisdicciones estatales se relacionaban con reconocer los repertorios mediante los cuales los barrios "habían sido escuchados". Sobre este punto, no encontramos prácticas innovadoras respecto de otras experiencias: los cortes de calle fueron la herramienta más usada, y a partir de ellos se obtuvieron reuniones con instituciones estatales provinciales y municipales.

Al describir el caso, si bien el acto de ocupar la tierra se dio ante una necesidad irresuelta, y fue a partir de ella que se dieron las negociaciones para comprar la tierra, en las entrevistas realizadas no se reconoció el hecho mismo de la ocupación como una instancia de reclamo. Ante la indagación sobre las formas que tomaron los reclamos hacia el Estado, se reconocen únicamente cuestiones ligadas a la urbanización (mejoramiento de calles, acceso a los servicios de agua y luz):

"Por cuestiones de la luz sí hicimos algunas marchas. También para el agua y no tuvimos respuesta. Pero por el terreno, no."(C., vecina de El Molino)

Lxs entrevistadxs indican que para acceder a la tierra hubo intervención estatal para mediar la venta con lxs propietarixs. Pero no reconocen en el acto de ocupación un acto de reclamo. Lo mismo sucede en el testimonio de una de las estudiantes de Arquitectura participante:

"Se negoció con una persona que estaba en Tierras en La Plata, bastante accesible en el sentido de que todas las partes salgan favorecidas, y bueno, no fue tanto conflicto el tema de la toma. Sí pedían que se regularice, o sea que sea ordenado el tema del asentamiento posterior." (V., estudiante de arquitectura entrevistada)

Los vínculos con funcionarixs públicxs conseguidos mediante reclamos fueron de gran importancia en las instancias de negociación. Sin embargo, en la vinculación con funcionarixs también fueron relevantes los contactos facilitados desde la organización social. Ambos elementos -los repertorios de reclamo y los contactos a través de organizaciones sociales- resultaron necesarios para iniciar y avanzar los procesos de regularización dominial. 


\section{Lo que la regularización dominial organizó}

Si bien el punto de partida de la investigación buscaba reconocer los aportes de la acción colectiva en los procesos de regularización dominial, el trabajo de campo nos llevó a encontrar que esa dimensión colectiva no se circunscribió a la regularización dominial, sino que también se fue "inoculando" en otros procesos de mejoramiento habitacional. Las prácticas colectivas se reflejaron en los procesos de ocupación de las tierras, de construcción de viviendas y de conexión a los servicios.

Tal como señala Di Virgiglio (2007), una vez resuelto el acceso al suelo, comienzan a atenderse otros aspectos de las estrategias habitacionales: la vivienda, la infraestructura, los equipamientos comunitarios. Como indicamos, la nacionalidad de origen resulta relevante: lxs vecinxs, lxs técnicxs y lxs integrantes de las organizaciones sociales entrevistadxs coinciden en que la mayoría de los migrantes paraguayxs y del norte de la Argentina son trabajadores de la construcción, por lo que al momento de construir los barrios y las viviendas contaron con conocimientos que se reflejan en los grados de consolidación de cada barrio. Además, cuentan con prácticas comunitarias propias de sus lugares de origen: en distintas instancias de construcción y mejoramiento barrial encontramos prácticas como la minga y los fondos comunitarios de dinero para compra de materiales para conexión de servicios. F. (vecino de El Molino) mencionaba al respecto:

"Nos ingeniamos también para construir las casas, sin ayuda de ningún arquitecto, de nadie."

En lo que se refiere a urbanización, El Molino presenta un grado de consolidación en sus viviendas, los espacios públicos, el equipamiento comunitario. Entre las explicaciones encontramos el hecho de que la actividad primaria de prácticamente todos sus habitantes varones es el trabajo en la construcción. Además, varias familias trabajan actualmente en cooperativas de trabajo coordinadas desde el FOL, parte de cuya tarea es mantenimiento y mejora del propio barrio. Si bien en el momento de la ocupación de este barrio se contó con asesoramiento del equipo técnico de Arquitectura, la frecuente inexistencia de asistencia técnica o asesoramiento sobre el tema en las instancias posteriores fue suplantada por los saberes de lxs habitantes del barrio, que fueron compartidos entre vecinxs.

En cuanto a la conexión a servicios básicos, todas las conexiones son autoproducidas. Luego de la toma, la conexión a la red de agua y al tendido eléctrico fue urgente. Tras presentar los correspondientes pedidos a las empresas prestatarias y no recibir respuestas, se realizaron jornadas colectivas de conexiones clandestinas a los servicios. La mayoría de las familias aportó dinero para la compra de cables y/o caños, y en jornadas de trabajo durante feriados o domingos realizaron las conexiones.

La continuidad en el trabajo territorial del FOL en El Molino, así como su crecimiento como movimiento social en los últimos años, se refleja en la cantidad de actividades que se desplegaron en ese barrio, ligadas al trabajo cooperativo. Semanas después de ocupar el predio, se decidió construir un comedor comunitario. Con la ayuda del Movimiento 7 de abril, se acordó en una de las asambleas que se realizaban los días sábados empezar a construir el Comedor Oga Guazú (Casa Grande en guaraní), donde funcionó durante 5 años el comedor barrial. Desde el año 2018 se empezó a construir en el mismo predio el nuevo comedor, en coordinación con las cooperativas de trabajo del FOL (en las que participan varixs vecinxs del barrio). Este espacio físico resultó de gran importancia para abordar algunos de los nuevos problemas que fueron surgiendo: con el tiempo aparecieron nuevos conflictos como alimento, trabajo, géneros, educación, seguridad, urbanización, entre otros. Estos temas fueron buscando respuestas en la asamblea barrial, que comenzó siendo del barrio El Molino y actualmente es del FOL-Villa Elvira. En el comedor funcionan la Asamblea, el Comedor comunitario, la Guardería (en horario de trabajo de lxs cooperativistas y durante las movilizaciones) y los Talleres de oficios, y se desarrollan las reuniones de la Cooperativa de Huerta y de la cooperativa de Construcción (trabajan en la plaza, donde tienen planificada una cancha de vóley, una de fútbol y parrillas), el Plan de Finalización de Estudios Primarios y Secundarios (FinES) y la fiesta de San Juan (tradicional de Paraguay). A través de la coordinación con otros espacios de trabajo del FOL (particularmente 
la cooperativa de herrería que funciona en el centro cultural y social Galpón de Tolosa), se realizó la señalética y se instalaron dos contenedores de basura.

Encontramos también otras instancias en las que, desde el deber ser de la acción colectiva, podrían haberse esperado abordajes colectivos, pero no se dieron. En los procesos de ocupación y urbanización, observamos prácticas colectivas que dieron legitimidad a la ocupación:

- tanto en el momento de la ocupación como en las prácticas cotidianas referidas a la seguridad en la tenencia de la tierra, se registran acciones que podrían interpretarse como "racionalidades normativas tácitas-sociales" (Rincón Patiño, 2006).

- en el proceso de autourbanización, los criterios adoptados para la distribución de lotes y la aprobación o desaprobación en asambleas de los cambios de ocupantes dan cuenta de prácticas comunitarias que buscaron la regulación a partir de normativas internas.

En la construcción de estas racionalidades normativas se incorporaron -o se aspira a incorporar-parámetros propios de la normatividad estatal (Rolnik, 1996; Azuela de la Cueva, 1993; Canestraro, 2010), con acciones y métodos colectivos en sus desarrollos.

Pero en el caso de la regularización dominial, se adoptan características exclusivamente de la normatividad estatal, sin incorporar necesariamente aspectos de las normativas sociales empleadas hasta entonces en el proceso de ocupación y urbanización. Si bien hasta ese momento la seguridad en la tenencia se fue dando mediante vínculos vecinales, la búsqueda de la regularización dominial (y con ello el reconocimiento estatal) no incorpora lógicas colectivas o innovadoras respecto de la forma que esa tenencia adopta, sino que retoma la única opción legal en oferta: la propiedad privada individual.

\section{REFLEXIONES}

A modo de síntesis podemos decir que en el caso analizado la regularización dominial avanzó hasta donde lo hizo gracias a los aportes surgidos de la coordinación y la gestación de vínculos entre vecinxs, organizaciones, estudiantes y funcionarixs públicxs. Sobre ello, nos interesa ahondar en tres reflexiones.

En primer lugar, a pesar de que los procesos de regularización dominial implican la participación estatal, en el caso analizado su llegada se dio a través de reclamos o contactos previos, y no como parte de acciones programadas. Asimismo, si bien los instrumentos de regularización utilizados tuvieron vinculación con la situación dominial previa, y hubo fuertes instancias de organización barrial, los procesos no culminaron, lo que deja expuestas las limitaciones de la política de regularización dominial.

En segundo lugar, si bien no era el objetivo original, los aportes de la acción colectiva no se limitaron a la ocupación de tierras y la regularización dominial, sino que tuvieron su influencia en otros procesos del barrio (como los referidos a acceso a servicios y equipamientos, espacios comunitarios, atención de conflictos de género, funcionamiento de programas educativos, etc.). Si bien cada una de las partes aportó algo y todos los aportes fueron relevantes, lxs vecinxs coinciden en la relevancia de la organización de los barrios. En las entrevistas encontramos la valoración de la organización barrial como elemento fundamental en los procesos de regularización dominial y el reconocimiento de aspectos positivos y negativos en las coordinaciones que se fueron dando. Lo que en todos los casos queda claro es el aspecto positivo de haberse organizado en la instancia de acceso a la tierra y en los procesos por la urbanización. Positivo en el sentido de haber resuelto luego de largas búsquedas el acceso a la tierra y la vivienda, con cierto grado de seguridad en la tenencia. Si bien la organización barrial no garantizó el avance del procedimiento de regularización dominial, encontramos que sí aportó características particulares para abordar el mejoramiento barrial.

Finalmente, ante la indagación específica sobre la valoración del acto colectivo como mecanismo de reclamo, no encontramos en las respuestas duda alguna sobre su relevancia. A pesar de que en los relatos de 
las entrevistas se pueden ir detectando conflictos, desgastes, distintos grados de involucramiento, disputas internas, la efectividad y la necesidad del acto colectivo son valoradas reiteradamente:

"La organización es buena, pero para que uno esté en la organización también tiene sus requisitos, uno tiene que estar preparado. Y no todos estamos preparados para estar dentro de una organización, porque somos tan individualistas" (F., entrevistado de El Molino).

En síntesis, reconociendo el condicionamiento de las coyunturas políticas, de las organizaciones intervinientes, de los contactos que puedan tenerse, la empiria y los relatos coinciden en que el aporte de la acción colectiva es un aspecto clave para la existencia y avance del mejoramiento barrial.

\section{REFERENCIAS}

Azuela de la Cueva, A. (1989). La ciudad, la propiedad y el derecho. México: Colegio de México.

Azuela de la Cueva, A. (1993). Los asentamientos populares y el orden jurídico en la urbanización periférica de América Latina. Revista Mexicana de Sociología, Volumen 55, N³, 133-168.

Baumann, F. (2002). Moradia e propriedade: um estudo sobre as percep̧̧óes de moradores e formuladores de politicas de habitação (Tesis en el Programa de Postgrado en Sociología). Universidad Federal de Rio Grande do Sul.

Canestraro, M. L. (2010). Entre el hecho y el derecho... De actores y prácticas en tres procesos de producción del hábitat (Mar del Plata, 1983-2009) (Tesis de Doctorado en Ciencias Sociales). Universidad de Buenos Aires. Facultad de Ciencias Sociales.

Del Río, J. P. y González, P. (2018). Los asentamientos populares informales en el Gran La Plata (pp.195-225). En Cravino, M. C. La ciudad (re)negada, Los Polvorines: Ediciones UNGS.

Di Croce Garay, A. y Alessio, A. (2018). Tomas de tierras en La Plata. Avances de un Registro de Tomas de Tierras para el período 2000-2015. Crítica y Resistencias. Revista de conflictos sociales latinoamericanos, №6, 18-46.

Di Croce Garay, A. y Doucet, D. (2019). Intervenciones estatales en las tomas de tierras. Análisis de las respuestas recibidas en los intentos de ocupaciones de tierras en La Plata durante el período 2004-2015. Ponencia presentada en II Jornadas de Sociología, UNMDP.

Di Virgiglio, M. (2007). Trayectorias residenciales y estrategias habitacionales de familias de sectores populares y medios en Buenos Aires (Tesis de Doctorado en Ciencias Sociales). Universidad de Buenos Aires. Facultad de Ciencias Sociales.

Herzer, H. y Pírez, P. (1993). Gestión urbana en ciudades intermedias de América Latina. Nairobi: UNCHS.

Piovani, J. I. (2007). La entrevista en profundidad. En A. Marradi, N. Archenti y J. I. Piovani (Eds.). Metodología de las ciencias sociales (pp. 215-225). Buenos Aires: Emecé.

Rincón Patiño, A. (2006). Racionalidades normativas y apropiación del territorio urbano: entre el territorio de la ley y la territorialidad de las legalidades. Revista Economía, Sociedad y Territorio, V(20), 673-702.

Rolnik, R. (1997). Legislación urbana y mercados informales de tierra en Sao Paulo, Brasil: el vínculo perverso. En Rolnik, R. A cidade e a lei: legislacao, politica urbana e territorios na cidade de Sao Paulo. San Pablo: Studio Nobel - Fapesp.

\section{FUENTES}

Censo Nacional de Población, Hogares y Vivienda 2010

Registro público Provincial de Villas y Asentamientos Precarios (RPPVAP)

Registro Nacional de Barrios populares (ReNaBaP)

Página web del FOL 


\section{Notas}

1. Tesis "Reconocer las resistencias. La dimensión colectiva en procesos de regularización dominial de asentamientos informales en La Plata”, de la Maestría en Estudios Urbanos de la UNGS. El artículo se enmarca en el Proyecto de $\mathrm{I}+\mathrm{D}+\mathrm{i}$ "Producción Social del Hábitat en áreas metropolitanas del Norte y el Sur Global: políticas, instituciones y movilización social” Ref.: (PID2019-105205RB-100), AEI:10.130.139/501100011033 Ministerio de Ciencia, Innovación y Universidades (España).

2. Siguiendo la definición del registro Nacional de Barrios Populares (RENABAP), se denomina Barrio Popular a los barrios vulnerables en los que viven al menos 8 familias agrupadas o contiguas, donde más de la mitad de la población no cuenta con título de propiedad del suelo ni acceso regular a dos, o más, de los servicios básicos (red de agua corriente, red de energía eléctrica con medidor domiciliario y/o red cloacal).

3 http://www.sstuv.gba.gov.ar/idehab/

4. Durante el primer semestre de 2019, luego de que algunxs vecinxs reclamaran, en el RENABAP se diferenció al barrio El Molino de Joel.

5. Como describen Del Río y González (2018), referirnos a formalidad e informalidad nos acerca a conceptos multidimensionales y controvertidos. Ambos aluden al cumplimiento (o no) de normativas que refieren a aspectos dominiales y/o urbanísticos: reglamentos de edificación, normativas de ocupación de suelo tramitación de permisos constructivos, declaración de las construcciones y mejoras, etc.

6. Expresiones registradas durante las entrevistas.

7. Esta experiencia fue expuesta en diversos plenarios del FOL y algunos de sus aspectos fueron publicados en la revista Tierra para vivir, de la misma organización. https://issuu.com/tierraparavivir

8. Como se describe en Di Croce Garay (2019), en las ocupaciones de tierras la forma de intervención estatal utilizada con mayor frecuencia es el desalojo, actos generalmente caracterizados por el uso de la violencia institucional y la omisión del diálogo.

9. En los relatos se menciona ese árbol como referencia del lugar de reunión, en el centro del predio ocupado.

10. Término registrado en varias ocupaciones de tierras, para referirse a la posibilidad de que otras personas ocupen el predio ya ocupado.

11. Publicado el 21/11/2016: https://www.facebook.com/FOLlaplataberisso/photos/a.632354313448255/1543717528 $978591 /$ ?type $=3 \&$ theater ( tomado el 25/06/2019).

12. Los asentamientos populares presentan una serie de diferencias morfológicas y urbanísticas respecto de las villas. Una de las principales diferencias se refiere a la continuidad de la trama urbana de la ciudad formal, con el objetivo posterior de regularizar el asentamiento. La expresión "Hacer una villa" se refiere a evitar irregularidades urbanas que a posteriori dificulten la regulación de la ocupación. 